\title{
ERRATUM OF ARTICLE "REDUCED-ORDER UNSCENTED KALMAN FILTERING WITH APPLICATION TO PARAMETER IDENTIFICATION IN LARGE-DIMENSIONAL SYSTEMS"
}

\section{Philippe Moireau ${ }^{1}$ And Dominique Chapelle ${ }^{1}$}

Received November 16, 2010.

Published online January 19, 2011.

\section{ESAIM: COCV (2010) DOI: 10.1051/cocv/2010006}

Some errors were introduced in (3.8) when summarizing the derivations of Section 3.2 (specifically in the second line of $(3.8 \mathrm{~b})$ and third line of $(3.8 \mathrm{c})$ ), hence we here rewrite the whole corrected summary for completeness.

Algorithm summary for the simplex case. Given adequate sampling rules, precompute the corresponding $\left[I^{*}\right]$,

- Sampling:

$\left\{\begin{array}{l}C_{n}=\sqrt{U_{n}^{-1}} \\ \hat{X}_{n}^{(i)+}=\hat{X}_{n}^{+}+L_{n} C_{n} I^{(i)}, \quad 1 \leq i \leq p+1 .\end{array}\right.$

- Prediction:

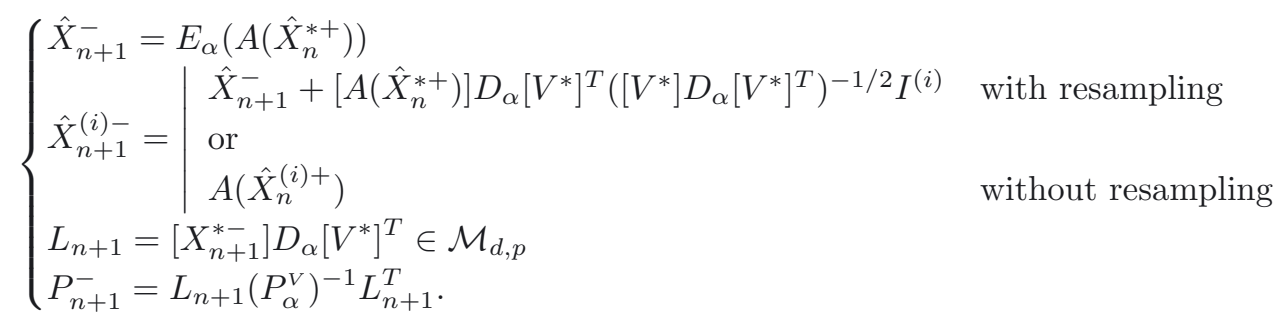

- Correction:

$$
\left\{\begin{array}{l}
Z_{n+1}^{(i)}=H\left(\hat{X}_{n+1}^{(i)-}\right), \\
\{H L\}_{n+1}=\left[Z_{n+1}^{*}\right] D_{\alpha}\left[V^{*}\right]^{T} \\
U_{n+1}=P_{\alpha}^{V}+\{H L\}_{n+1}^{T} W_{n+1}^{-1}\{H L\}_{n+1} \in \mathcal{M}_{p} \\
\hat{X}_{n+1}^{+}=\hat{X}_{n+1}^{-}+L_{n+1} U_{n+1}^{-1}\{H L\}_{n+1}^{T} W_{n+1}^{-1}\left(Z_{n+1}-E_{\alpha}\left(Z_{n+1}^{*}\right)\right) \\
P_{n+1}^{+}=L_{n+1} U_{n+1}^{-1} L_{n+1}^{T} .
\end{array}\right.
$$

${ }^{1}$ INRIA, B.P. 105, 78153 Le Chesnay Cedex, France. philippe.moireau@inria.fr; dominique.chapelle@inria.fr 
Next, although the other algorithm summary (3.14) was algebraically correct, it implicitly assumed the matrix $D_{\mathfrak{m}}$ to be invertible, which does not always hold. Hence, we rewrite the whole algorithm without using this assumption.

Algorithm summary for the general case. Given adequate sampling rules, precompute the correspond$\operatorname{ing}\left[V^{*}\right], P_{\alpha}^{V}=\left[V^{*}\right] D_{\alpha}\left[V^{*}\right]^{T},\left[I^{*}\right]=\left(\left[V^{*}\right] D_{\alpha}\left[V^{*}\right]^{T}\right)^{-\frac{1}{2}}\left[V^{*}\right]$, and $D_{V}=D_{\alpha}\left[V^{*}\right]^{T}\left(P_{\alpha}^{V}\right)^{-1}\left[V^{*}\right] D_{\alpha}$.

- Sampling:

$\left\{\begin{array}{l}C_{n}=\sqrt{U_{n}^{-1}} \\ \hat{X}_{n}^{(i)+}=\hat{X}_{n}^{+}+L_{n} C_{n} I^{(i)}, \quad 1 \leq i \leq r .\end{array}\right.$

- Prediction:

$$
\left\{\begin{array}{l}
\hat{X}_{n+1}^{-}=E_{\alpha}\left(A\left(\hat{X}_{n}^{*+}\right)\right) \\
\hat{X}_{n+1}^{(i)-}=\hat{X}_{n+1}^{-}+\left[A\left(\hat{X}_{n}^{*+}\right)-\hat{X}_{n+1}^{-}\right] D_{\alpha}^{\frac{1}{2}} \Upsilon_{p} I^{(i)}, \quad \text { resampling with SVD } \\
L_{n+1}=\left[X_{n+1}^{*-}\right] D_{\alpha}\left[V^{*}\right]^{T} \in \mathcal{M}_{d, p} \\
P_{n+1}^{-}=L_{n+1}\left(P_{\alpha}^{V}\right)^{-1} L_{n+1}^{T} .
\end{array}\right.
$$

\section{- Correction:}

$$
\left\{\begin{array}{l}
{[\tilde{Z}]=\left[H\left(\hat{X}_{n+1}^{*}\right)-E_{\alpha}\left(H\left(\hat{X}_{n+1}^{*}\right)\right)\right]} \\
D_{\mathfrak{m}}=[\tilde{Z}]^{T} W_{n+1}^{-1}[\tilde{Z}] \in \mathcal{M}_{r} \\
U_{n+1}=P_{\alpha}^{V}+\left[V^{*}\right] D_{\alpha}\left(\mathbb{1}+D_{\mathfrak{m}}\left(D_{\alpha}-D_{V}\right)\right)^{-1} D_{\mathfrak{m}} D_{\alpha}\left[V^{*}\right]^{T} \in \mathcal{M}_{p} \\
\{H L\}_{n+1}=[\tilde{Z}]\left(\mathbb{1}+D_{\alpha} D_{\mathfrak{m}}\right)^{-1}\left(\mathbb{1}+D_{V}\left(\mathbb{1}+D_{\mathfrak{m}}\left(D_{\alpha}-D_{V}\right)\right)^{-1} D_{\mathfrak{m}}\right) D_{\alpha}\left[V^{*}\right]^{T} \\
\hat{X}_{n+1}^{+}=\hat{X}_{n+1}^{-}+L_{n+1} U_{n+1}^{-1}\{H L\}_{n+1}^{T} W_{n+1}^{-1}\left(Z_{n+1}-E_{\alpha}\left(Z_{n+1}^{*}\right)\right) \\
P_{n+1}^{+}=L_{n+1} U_{n+1}^{-1} L_{n+1}^{T} .
\end{array}\right.
$$

We provide the proof for the correction step (3.14c) which contains the alternative equations valid without any assumption on $D_{\mathfrak{m}}$. First, we can write the filter in the form

$$
\hat{K}_{n+1}=P_{\alpha}^{\tilde{X} \tilde{z}}\left(P_{\alpha}^{\tilde{z}}\right)^{-1}
$$

and we will compute this operator using the matrix inversion lemma to obtain a tractable algorithm. To this end we introduce the following compact notation

$$
[\tilde{X}]=\left[\hat{X}_{n+1}^{*}-\hat{X}_{n+1}^{-}\right], \quad[\tilde{Z}]=\left[Z_{n+1}^{*}-E_{\alpha}\left(Z_{n+1}^{*}\right)\right]
$$

and we then have

$$
\begin{aligned}
\hat{K}_{n+1} & =[\tilde{X}] D_{\alpha}[\tilde{Z}]^{T}\left(W_{n+1}+[\tilde{Z}] D_{\alpha}[\tilde{Z}]^{T}\right)^{-1} \\
& =[\tilde{X}] D_{\alpha}[\tilde{Z}]^{T}\left(W_{n+1}^{-1}-W_{n+1}^{-1}[\tilde{Z}]\left(D_{\alpha}^{-1}+[\tilde{Z}]^{T} W_{n+1}^{-1}[\tilde{Z}]\right)^{-1}[\tilde{Z}]^{T} W_{n+1}^{-1}\right) \\
& =[\tilde{X}] D_{\alpha}\left(\mathbb{1}_{r}-[\tilde{Z}]^{T} W_{n+1}^{-1}[\tilde{Z}]\left(D_{\alpha}^{-1}+[\tilde{Z}]^{T} W_{n+1}^{-1}[\tilde{Z}]\right)^{-1}\right)[\tilde{Z}]^{T} W_{n+1}^{-1}
\end{aligned}
$$

Let us now set

$$
D_{\mathfrak{m}}=[\tilde{Z}]^{T} W_{n+1}^{-1}[\tilde{Z}] \in \mathcal{M}_{r},
$$

which - unlike for $P_{\alpha}^{\tilde{Z}}$ - can be computed in practice, since its dimension is equal to the number of sigma-points. 
We thus have

$$
\begin{aligned}
\hat{K}_{n+1} & =[\tilde{X}] D_{\alpha}\left(\mathbb{1}-D_{\mathfrak{m}}\left(D_{\alpha}^{-1}+D_{\mathfrak{m}}\right)^{-1}\right)[\tilde{Z}]^{T} W_{n+1}^{-1}, \\
& =[\tilde{X}] D_{\alpha}\left(\mathbb{1}+D_{\mathfrak{m}} D_{\alpha}\right)^{-1}[\tilde{Z}]^{T} W_{n+1}^{-1},
\end{aligned}
$$

where we have used the matrix inversion lemma in the second line. Note that the invertibility of a matrix $\mathbb{1}+A B$ with both $A$ and $B$ symmetric positive matrices is a standard property (e.g. one-to-one can be proven by decomposing $\mathbb{R}^{r}$ into the direct sum of $\operatorname{Ker} A$ and $\operatorname{Im} A$ ). Then, by the same argument as in Proposition 3.1 , the filter can also be written in the form

$$
\hat{K}_{n+1}=L_{n+1}\left(P_{\alpha}^{V}\right)^{-1}\left[V^{*}\right] D_{\alpha}\left(\mathbb{1}+D_{\mathfrak{m}} D_{\alpha}\right)^{-1}[\tilde{Z}]^{T} W_{n+1}^{-1},
$$

with

$$
L_{n+1}=\left[\hat{X}_{n+1}^{*-}\right] D_{\alpha}\left[V^{*}\right]^{T} .
$$

Note that the term $[\tilde{Z}]^{T}$ in (1) cannot be treated in the same manner since the sigma-points propagated by the observation operator do not satisfy the original constraints. In addition to the gain, we also need to compute the a posteriori covariance matrix in order to resample at the next step. We have

$$
\begin{aligned}
P_{n+1}^{+} & =P_{n+1}^{-}-P_{\alpha}^{\tilde{X} \tilde{z}}\left(P_{\alpha}^{\tilde{z}}\right)^{-1}\left(P_{\alpha}^{\tilde{X} \tilde{z}}\right)^{T} \\
& =P_{n+1}^{-}-[\tilde{X}] D_{\alpha}\left(\mathbb{1}-D_{\mathfrak{m}}\left(D_{\alpha}^{-1}+D_{\mathfrak{m}}\right)^{-1}\right) D_{\mathfrak{m}} D_{\alpha}[\tilde{X}]^{T}
\end{aligned}
$$

We now use the matrix inversion lemma as in (1) to simplify

$$
\begin{aligned}
P_{n+1}^{+} & =P_{n+1}^{-}-[\tilde{X}] D_{\alpha}\left(\mathbb{1}+D_{\mathfrak{m}} D_{\alpha}\right)^{-1} D_{\mathfrak{m}} D_{\alpha}[\tilde{X}]^{T} \\
& =P_{n+1}^{-}-L_{n+1}\left(P_{\alpha}^{V}\right)^{-1}\left[V^{*}\right] D_{\alpha}\left(\mathbb{1}+D_{\mathfrak{m}} D_{\alpha}\right)^{-1} D_{\mathfrak{m}} D_{\alpha}\left[V^{*}\right]^{T}\left(P_{\alpha}^{V}\right)^{-1} L_{n+1}^{T} \\
& =L_{n+1}\left(\left(P_{\alpha}^{V}\right)^{-1}-\left(P_{\alpha}^{V}\right)^{-1}\left[V^{*}\right] D_{\alpha}\left(\mathbb{1}+D_{\mathfrak{m}} D_{\alpha}\right)^{-1} D_{\mathfrak{m}} D_{\alpha}\left[V^{*}\right]^{T}\left(P_{\alpha}^{V}\right)^{-1}\right) L_{n+1}^{T} .
\end{aligned}
$$

The advantage of this last form is that we can again write

$$
P_{n+1}^{+}=L_{n+1} U_{n+1}^{-1} L_{n+1}^{T},
$$

with

$$
U_{n+1}^{-1}=\left(P_{\alpha}^{V}\right)^{-1}-\left(P_{\alpha}^{V}\right)^{-1}\left[V^{*}\right] D_{\alpha}\left(\mathbb{1}+D_{\mathfrak{m}} D_{\alpha}\right)^{-1} D_{\mathfrak{m}} D_{\alpha}\left[V^{*}\right]^{T}\left(P_{\alpha}^{V}\right)^{-1} .
$$

Hence, defining $D_{V} \in \mathcal{M}_{r}$ as

$$
D_{V}=D_{\alpha}\left[V^{*}\right]^{T}\left(P_{\alpha}^{V}\right)^{-1}\left[V^{*}\right] D_{\alpha},
$$

we can simplify - with another application of the matrix inversion lemma

$$
U_{n+1}=P_{\alpha}^{V}+\left[V^{*}\right] D_{\alpha}\left(\mathbb{1}+D_{\mathfrak{m}}\left(D_{\alpha}-D_{V}\right)\right)^{-1} D_{\mathfrak{m}} D_{\alpha}\left[V^{*}\right]^{T}
$$

This identity of course requires that $\left(\mathbb{1}+D_{\mathfrak{m}}\left(D_{\alpha}-D_{V}\right)\right)$ be invertible, which can be established by proving that $D_{\alpha}-D_{V}$ is a symmetric positive matrix. We have by definition, indeed,

$$
\left[V^{*}\right] D_{V}\left[V^{*}\right]^{T}=P_{\alpha}^{V}\left(P_{\alpha}^{V}\right)^{-1} P_{\alpha}^{V}=\left[V^{*}\right] D_{\alpha}\left[V^{*}\right]^{T}
$$

hence, for any vector $R \in \mathbb{R}^{r}$ in the range of the rows of $\left[V^{*}\right]$

$$
R^{T}\left(D_{\alpha}-D_{V}\right) R=0 .
$$


If we now consider $S \in \mathbb{R}^{r} D_{\alpha}$-orthogonal to this range, namely, satisfying

$$
\left[V^{*}\right] D_{\alpha} S=0
$$

we have

$$
S^{T} D_{V} S=S^{T} D_{\alpha}\left[V^{*}\right]^{T}\left(P_{\alpha}^{V}\right)^{-1}\left[V^{*}\right] D_{\alpha} S=0
$$

hence,

$$
S^{T}\left(D_{\alpha}-D_{V}\right) S=S^{T} D_{\alpha} S \geq 0
$$

which shows that $D_{\alpha}-D_{V}$ is positive as claimed.

It is now obvious that by defining

$$
\{H L\}_{n+1}=[\tilde{Z}]\left(\mathbb{1}+D_{\alpha} D_{\mathfrak{m}}\right)^{-1}\left(\mathbb{1}+D_{V}\left(\mathbb{1}+D_{\mathfrak{m}}\left(D_{\alpha}-D_{V}\right)\right)^{-1} D_{\mathfrak{m}}\right) D_{\alpha}\left[V^{*}\right]^{T},
$$

we can rewrite the filter in the following form

$$
\hat{X}_{n+1}^{+}=\hat{X}_{n+1}^{-}+L_{n+1} U_{n+1}^{-1}\{H L\}_{n+1}^{T} W_{n+1}^{-1}\left(Z_{n+1}-E_{\alpha}\left(Z_{n+1}^{*}\right)\right) .
$$

Finally, we point out that this algorithm is implemented in the Verdandi ${ }^{2}$ opensource data assimilation library.

\footnotetext{
${ }^{2}$ http://verdandi.gforge.inria.fr/
} 